\title{
On groups of formal diffeomorphisms of several complex variables
}

\author{
MITCHAEL MARTELO ${ }^{1}$ and BRUNO SCÁRDUA ${ }^{2}$ \\ ${ }^{1}$ Instituto de Matemática, Universidade Federal Fluminense, Departamento de Matemática Aplicada (GMA), \\ Rua Mário Santos Braga, s/n, 24010-140 Niterói, RJ, Brasil \\ ${ }^{2}$ Instituto de Matemática, Universidade Federal do Rio de Janeiro, \\ Caixa Postal 68530, 21945-970 Rio de Janeiro, RJ, Brasil
}

Manuscript received on July 7, 2011; accepted for publication on March 19, 2012

\begin{abstract}
In this note we announce some results in the study of groups of formal or germs of analytic diffeomorphisms in several complex variables. Such groups are related to the study of the transverse structure and dynamics of Holomorphic foliations, via the holonomy group notion of a foliation's leaf. For dimension one, there is a well-established dictionary relating analytic/formal classification of the group, with its algebraic properties (finiteness, commutativity, solvability, among others). Such system of equivalences also characterizes the existence of suitable integrating factors, i.e., invariant vector fields and one-forms associated to the group. Our aim is to state the basic lines of such dictionary for the case of several complex variables groups. Our results are applicable in the construction of suitable integrating factors for holomorphic foliations with singularities. We believe they are a starting point in the study of the connection between Liouvillian integration and transverse structures of holomorphic foliations with singularities in the case of arbitrary codimension. The results in this note are derived from the $\mathrm{PhD}$ thesis "Grupos de germes de difeomorfismos complexos em várias variáveis e formas diferenciais" of the first named author (Martelo 2010).
\end{abstract}

Key words: Formal complex diffeomorphism, infinitesimal generator, holomorphic foliation, germ of complex diffeomorphism.

\section{INTRODUCTION}

The study of groups and germs of complex diffeomorphisms fixing the origin is an important tool in Complex Dynamics and in the theory of Holomorphic Foliations via the study of holonomy groups (Camacho and Lins Neto 1985) of its leaves. Indeed, the holonomy groups (of the leaves) of a codimension $n \geq 1$ holomorphic foliation are

AMS Classification Primary 32S65; Secondary 57R30.

Correspondence to: Mitchael Martelo

E-mail: mitchaelmartelo@id.uff.br (identified with) groups of germs of complex diffeomorphisms fixing the origin of $\mathbb{C}^{n}$. In the codimension $n=1$, case these are subgroups of germs of one variable holomorphic maps and there is a wellestablished dictionary relating topological and dynamical properties of (the leaves of) the foliation to algebraic properties of the group. This is clear in works as (Camacho et al 1992), (Il'yashenko 1978), (Nakai 1994) and (Scherbakov 2006). All these facts are compiled in some works relating the existence of suitable "transverse structures" for the foliation with 
the transverse dynamics of the foliation (Camacho and Scárdua 2001), (Scárdua 1997, 1999). In (Martinez 2003), F. Brochero studies groups of germs of complex analytic diffeomorphisms having a fixed point at the origin. For such groups the author gives a nice study mainly focused on the analytical or topological description of the following cases: finite groups, linearizable groups, abelian groups containing a "generic" dicritic diffeomorphism. This important work also motivates some of the concepts and results in our work.

We study groups of formal or germs of analytic diffeomorphisms in several complex variables. Such groups are related to the study of the transverse structure and dynamics of Holomorphic foliations, via the notion of holonomy group of a leaf of a foliation. For dimension one, there is a well-established dictionary relating analytic/ formal classification of the group, with its algebraic properties (finiteness, commutativity, solvability, among others). Such system of equivalences also characterizes the existence of suitable integrating factors, i.e., invariant vector fields and oneforms associated to the group. In this research announcement, we explain the main results from (Martelo and Scárdua 2011), where we search the basic lines of such dictionary for the case of several complex variables groups. For abelian, metabelian, solvable or nilpotent groups we investigate the existence of suitable formal vector fields and closed differential forms which exhibit an invariance property under the group action. Our results are applicable in the construction of suitable integrating factors for holomorphic foliations with singularities. We believe they are a starting point in the study of the connection between Liouvillian integration and transverse structures of holomorphic foliations with singularities in the case of arbitrary codimension.

\section{PRELIMINARIES, NOTATION AND DEFINITIONS}

Let us introduce the notation we use throughout this research announcement. We denote by $\mathcal{O}_{n}$ the ring of germs at the origin of holomorphic functions of $n$ variables and by $\hat{\mathcal{O}}_{n}$ its formal completion, see (Eisenbud 1995) for a detailed construction of the formal completion of the ring $\mathcal{O}_{n}$, as well as for some classical concepts related to the formal objects we deal with in this announcement). Denote by $\operatorname{Diff}\left(\mathbb{C}^{n}, 0\right)$ the group of germs of complex diffeomorphisms fixing the origin $0 \in \mathbb{C}^{n}$. We denote by $z=\left(z_{1}, \ldots, z_{n}\right)$ a system of complex variables in $\mathbb{C}^{n}$. The group of formal diffeomorphisms in $\mathrm{n}$ complex variables fixing the origin is the formal completion Diff $\left(\mathbb{C}^{n}, 0\right)$ of $\operatorname{Diff}\left(\mathbb{C}^{n}, 0\right)$, obtained from the power series of the coordinate functions of elements in $\operatorname{Diff}\left(\mathbb{C}^{n}, 0\right)$. This way, given a formal diffeomorphism $f^{\prime} \in \operatorname{Diff}\left(\mathbb{C}^{n}\right.$, $0)$ we write $\hat{f}=\hat{f}^{\prime}(0) \cdot z+\sum_{j=2}^{\infty} f_{j}(z)$ where $\hat{f}^{\prime}(0) \in$ $\operatorname{GL}(n, \mathbb{C})$ and each $f_{j}$ is a vector whose coordinates are homogeneous polynomials of degree $j$ in the variables $z=\left(z_{1}, \ldots, z_{n}\right)$. To each formal diffeomorphism $f \in \operatorname{Diff}\left(\mathbb{C}^{n}, 0\right)$ we associate its derivative $\hat{f}^{\prime}(0) \in \mathrm{GL}(n, \mathbb{C})$. We say that the diffeomorphism $\hat{f}$ is tangent to the identity if $\hat{f}$ $'(0)=$ Id. Denote by $\operatorname{Diff}_{\text {Id }}\left(\mathbb{C}^{n}, 0\right)$ the subgroup of elements tangent to the identity in $\operatorname{Diff}\left(\mathbb{C}^{n}, 0\right)$. Also put Diff $\operatorname{Id}_{\text {Id }}\left(\mathbb{C}^{n}, 0\right)=\operatorname{Diff}_{\text {Id }}\left(\mathbb{C}^{n}, 0\right) \cap$ Diff $\left(\mathbb{C}^{n}, 0\right)$. This gives inclusions of $\operatorname{Diff}\left(\mathbb{C}^{n}, 0\right), \rightarrow$ $\operatorname{Diff}\left(\mathbb{C}^{n}, 0\right)$ and $\operatorname{Diff}_{\text {Id }}\left(\mathbb{C}^{n}, 0\right), \rightarrow \operatorname{Diff}_{\text {Id }}\left(\mathbb{C}^{n}, 0\right)$. Given a subgroup $G \subset \operatorname{Diff}\left(\mathbb{C}^{n}, 0\right)$ the linear part group is the subgroup $D G \subset \operatorname{GL}(n, \mathrm{C})$ defined by $D G=\left\{\hat{g}^{\prime}(0): \hat{g} \in G\right\}$. A subgroup $G \subset \operatorname{Diff}_{\text {Id }}\left(\mathbb{C}^{n}, 0\right)$ is called tangent to the identity. The subgroup of formal diffeomorphisms, tangent to the identity with order $k$, is defined as $\operatorname{Diff}_{k}\left(\mathbb{C}^{n}, 0\right)=\left\{\hat{f} \in \operatorname{Diff}\left(\mathbb{C}^{n}\right.\right.$, 0) $\left.\mid \hat{f}(z)=z+f_{k}+{ }_{1}(z)+f_{k}+{ }_{2}(z)+\cdots, f_{k}+1 \neq 0\right\}$. Similar to the group of germs of holomorphic diffeomorphisms at the origin $0 \in \mathbb{C}^{n}$, tangent to the identity with order $k$ is defined as $\operatorname{Diff}_{k}\left(\mathbb{C}^{n}\right.$, $0)=\operatorname{Diff}_{k}\left(\mathbb{C}^{n}, 0\right) \cap \operatorname{Diff}\left(\mathbb{C}^{n}, 0\right)$. We denote by $\hat{K}_{n}$ the field of rational functions of $n$-complex variables and by $\hat{K}_{n}$ its formal counterpart, i.e., the field of fractions of the ring of formal power series $\hat{\mathcal{O}}_{n}=\mathbb{C}\left[\left[z_{1}, \ldots, z_{n}\right]\right]$. 


\section{FORMAL VECTOR FIELDS AND FORMAL DIFFEOMORPHISMS}

Denote by $\mathcal{X}\left(\mathbb{C}^{n}, 0\right)$ the $\mathcal{O}_{n}$-module of germs of complex vector fields vanishing at the origin $0 \in$ $\mathbb{C}^{n}$ and by $\hat{\mathcal{X}}\left(\mathbb{C}^{n}, 0\right)$ its formal counterpart. The Lie algebra $\hat{\mathcal{X}}_{k}\left(\mathbb{C}^{n}, 0\right)$ of formal vectors fields of $\mathbb{C}^{n}$ of order $k+1$ is defined by those vector fields for the form $\hat{X}=\hat{a}_{1}(z) \frac{\partial}{\partial z 1}+\cdots+\hat{a}_{n}(z) \frac{\partial}{\partial z_{n}} ; \hat{a}_{j} \in \hat{\mathcal{O}}_{n}$, where the minimum order of vanishing of the $\hat{a}_{j}$ at the origin is $k+1$. Thus, $\hat{\mathcal{X}}_{k}\left(\mathbb{C}^{n}, 0\right)$ is the formal completion of $\mathcal{X}_{k}\left(\mathbb{C}^{n}, 0\right)$; the set of germs of complex analytic vector fields which are singular of order $k+1$ at 0 .

The expression $\exp (t \hat{X})=\left(\sum_{j=0}^{\infty} \frac{t j}{j !} \hat{X}^{j}\left(z_{1}\right), \ldots\right.$, $\left.\sum_{j=0}^{\infty} \frac{t j}{j !} \hat{X}^{j}\left(z_{n}\right)\right)$ defines the exponential of $t \hat{X}$ for $\hat{X}$ $\in \hat{\mathcal{X}}\left(\mathbb{C}^{n}, 0\right)$ and $t \in \mathbb{C}$. Let us remark that $\hat{X}^{j}(g)$ is the result of applying $j$ times the derivation $\hat{X}$ to the power series $g$. The definition coincides with the classical one if $\hat{X}$ is a germ of convergent vector field. According to (Ilyashenko and Yakovenko 2007) the exponential map exp: $\hat{\mathcal{X}}_{k}\left(\mathbb{C}^{n}, 0\right) \rightarrow \operatorname{Diff}_{k}\left(\mathbb{C}^{n}, 0\right)$ is a bijection.

Definition 0.1 Let $\hat{f} \in \operatorname{Diff}_{k}\left(\mathbb{C}^{n}, 0\right)$. We denote by $\log \hat{f}$ the unique element of $\hat{\mathcal{X}}_{k}(\mathbb{C}, 0)$ such that $\hat{f}$ $=\exp (\log \hat{f})$. We say that $\log \hat{f}$ is the infinitesimal generator of $\hat{f}$. Given a map $\hat{h} \in \operatorname{Diff}_{\text {id }}\left(\mathbb{C}^{n}, 0\right)$ and $t$ $\in \mathbb{C}$ we denote by $\hat{h}^{[t]}=\exp (t . \hat{X})$ where $\hat{h}=\exp \hat{X}$.

\section{Solvable Length of A Lie AlgEBRA}

Let $\mathfrak{g}$ be a complex Lie algebra with Lie bracket of two elements $X, Y \in \mathfrak{g}$ denoted by $[X, Y] \in \mathfrak{g}$. Given Lie subalgebras $\mathfrak{h}$ and $\mathfrak{l}$ of $\mathfrak{g}$. We define $[\mathfrak{h}, \mathfrak{l}]$ the Lie subalgebra of $\mathfrak{g}$ generated by the elements of the form $[X, Y]$ for $X \in \mathfrak{h}$ and $Y \in \mathfrak{l}$. We define $\mathfrak{g}(0)=\mathfrak{g}, \mathfrak{g}(n+1)=\left[\mathfrak{g}^{(n)}, \mathfrak{g}^{(n)}\right] \forall \mathrm{n} \geq 0$, the derived series of $\mathfrak{g}$. We also define $\mathcal{C}^{0} \mathfrak{g}=\mathfrak{g}, \mathcal{C}^{n+1} \mathfrak{g}=\left[\mathfrak{g}, \mathcal{C}^{n} \mathfrak{g}\right]$ $\forall n \geq 0$ the descending central series of $\mathfrak{g}$.

Definition 0.2 (solvable length of a Lie algebra, cf. (Martelo and Ribon in press)). Let $\mathfrak{g}$ be a Lie algebra. We define $l(\mathfrak{g})$ the soluble length of $\mathfrak{g}$ as $l(\mathfrak{g})$ $=\min \left\{k \in \mathbb{N} \cup\{0\}: \mathfrak{g}^{(\mathrm{k})}=\{\operatorname{Id}\}\right\}$, where $\min \emptyset=\infty$. We say that $\mathfrak{g}$ is solvable if $l(\mathfrak{g})<\infty$. The Lie algebra $\mathfrak{g}$ is nilpotent if there exists $j \geq 0$ such that $C^{j} \mathfrak{g}$ $=\{$ Id $\}$. If $j$ is the minimum non-negative integer number with such a property we say that $\mathfrak{g}$ is of nilpotent class $j$.

\section{LIE AlGEBRA OF A GROUP OF DIFFEOMORPHISMS}

As in the case of Lie groups we can relate properties of a subgroup $G \subset \operatorname{Diff}\left(\mathbb{C}^{n}, 0\right)$ to properties of a certain Lie algebra associate to the group. Let us first explain how to construct such Lie algebra. The first step is to define the Lie subalgebra of $\hat{X}\left(\mathbb{C}^{n}, 0\right)$ associate to a subgroup $G$ of $\subset \operatorname{Diff}\left(\mathbb{C}^{n}, 0\right)$.

Definition 0.3 (cf. (Martelo and Ribon in press) Definition 3.8). Let $G$ a subgroup of $\operatorname{Diff}\left(\mathbb{C}^{n}, 0\right)$. A complex Lie subalgebra $\mathfrak{g}$ of $\hat{\mathcal{X}}\left(\mathbb{C}^{n}, 0\right)$ is the Lie algebra of $G$ if $\mathfrak{g}=\left\{\hat{X} \in \hat{\mathcal{X}}\left(\mathbb{C}^{n}, 0\right): \exp (t \hat{X}) \in\right.$ $G, \forall t \in \mathbb{C}\}$.

Though this definition seems quite natural, it can give few information about the group. Indeed, the Lie algebra of a discrete group is always $\{0\}$. Thus, we must construct a more rich algebra in this case, this construction is found in (Martelo and Ribon in press). Indeed, when the linear part group $D G$ is connected, we can build $\bar{G} \supset G$ a new group with the same algebraic properties of G. In particular, as for the solvability length, we have $l(\bar{G})=l(G)$. Roughly speaking $G$ is the algebraic closure of $G$, it has a natural Lie algebra $\mathfrak{g} \neq\{0\}$ and $l(\mathfrak{g})=l(G)$, see (Martelo and Ribon in press).

In our work, we are mainly interested in studying groups of diffeomorphisms tangent to the identity, that is subgroups $G \subset \operatorname{Diff}\left(\mathbb{C}^{n}, 0\right)$ with $D G=\{\operatorname{Id}\}$. Therefore, we may perform the above construction and we have the following definition:

Definition 0.4 (associate Lie algebra). Let $G$ be a subgroup of $\operatorname{Diff}\left(\mathbb{C}^{n}, 0\right)$. A complex Lie subalgebra $\mathfrak{g}$ of $\hat{\mathcal{X}}\left(\mathbb{C}^{n}, 0\right)$ is the "associate" Lie algebra of $G$, if $\mathfrak{g}$ is the Lie algebra of $\bar{G}$. By dimension of the associate Lie algebra we shall mean the dimension of Lie algebra g, viewed as vector space over $\hat{K}_{n}$. 
THE One-Dimensional CASE

The construction of a dictionary relating algebraic, dynamical and analytic properties of subgroups of diffeomorphisms is a very important tool in Complex Dynamics and Holomorphic foliations.

Definition 0.5. Given a subgroup $G \subset \operatorname{Diff}\left(\mathbb{C}^{n}, 0\right)$, a formal vector field $\hat{X} \in \hat{X}\left(\mathbb{C}^{n}, 0\right)$ is $G$-invariant if we have $\hat{g}_{*} \hat{X}=\hat{X}, \forall \hat{g} \in G$. We say that $\hat{X}$ is projectively invariant by $G$ if for each $\hat{g} \in G$ there is $c_{g} \in \mathbb{C}$ such that $\hat{g}_{*} \hat{X}=c_{\hat{g}} \cdot \hat{X}$.

Below we find a global picture of the onedimensional case:

Theorem 0.6 (One-dimensional case, (Loray 1994), (Il'yashenko and Yakovenko 2007)). Let $G$ $\subset \operatorname{Diff}(\mathbb{C}, 0)$ be a subgroup of one-variable formal complex diffeomorphisms.

1. $G$ is abelian if, and only if, $G$ is nilpotent if, and only if, $G$ admits a formal invariant vector field.

2. The following conditions are equivalent:

(a) $G$ is solvable.

(b) $G$ is metabelian, i.e., its subgroup of commutators [G,G] is abelian.

(c) All elements tangent to the identity in

$G$ have a same order of tangency.

(d) G admits a formal vector field which is projectively invariant by $G$.

Item (1) above is essentially a consequence of two other facts:

(i) For a subgroup $G \subset \operatorname{Diff}(\mathbb{C}, 0)$, the linear part group $D G=\left\{\hat{g}^{\prime}(0): \hat{g} \in \mathrm{G}\right\}$ is abelian and therefore the subgroup of commutators $[G, G] \subset G$, which is the set of products of commutators in $G$, is tangent to the identity.

(ii) Two elements $\hat{f}=z+a_{k}+1 z^{k+1}+\cdots \in G_{\mathrm{Id}}$, and $\hat{g}=z+b_{\ell+1} z^{\ell+1}+\cdots \in G_{\text {Id }}, a_{k}+1 . b_{\ell}+1 \neq 0$, tangent to the identity, commute only if we have $k=\ell$.
According to (Martelo and Scárdua 2011), none of the above facts holds for subgroups of $\operatorname{Diff}\left(\mathbb{C}^{n}, 0\right)$ when $n \geq 2$. Therefore it is quite natural to expect that the above mentioned dictionary is much different or much harder to find, in the $n \geq 2$ case. To begin this study is one of the main goals of this work.

\section{ABELIAN AND METABELIAN GROUPS}

Initially, we focus on the study of the associate Lie algebra of subgroups $G \subset \operatorname{Diff}\left(\mathbb{C}^{n}, 0\right)$ under the hypothesis that $G$ is abelian, metabelian or nilpotent. In (Martelo and Scárdua 2011) we prove the following (compare with (1) in Theorem 0.6):

Theorem 0.7 (Existence of an invariant formal vector field). Let $G \subset \operatorname{Diff}\left(\mathbb{C}^{n}, 0\right)$ be an abelian subgroup of formal diffeomorphisms. We have two possibilities:

1. G admits an invariant formal vector field.

2. $G \subset \operatorname{Diff}_{\mathrm{Id}}\left(\mathbb{C}^{n}, 0\right)=\{\mathrm{Id}\}$.

Regarding to the one-dimensional case we observe:

(i) Unlike the one-dimensional case, in general the existence of such an invariant vector field is not enough to assure that the group is abelian, see (Martelo and Scárdua 2011).

(ii) For $n=1$, condition (2) above implies that the group is conjugated to its linear part by a formal diffeomorphism. We believe that this also holds for dimension $n \geq 2$.

ABELIAN Subgroups AND NiLPOTENT SubALGEBRAS

In (Martinez 2003) it is proved (Proposition 4.1) that every nilpotent subalgebra $\mathcal{L}$ of $\hat{\mathcal{X}}\left(\mathbb{C}^{2}, 0\right)$ is metabelian. In (Martelo and Scárdua 2011) we prove, with an alternative proof, an extension of this result for higher dimension $n \geq 2$ :

Theorem 0.8. Every nilpotent subalgebra $\mathfrak{l}$ of $\hat{\mathcal{X}}$ $\left(\mathbb{C}^{n}, 0\right)$ has length l(1) at most $n$. As a consequence, if $G \subset \operatorname{Diff}_{\text {Id }}\left(\mathbb{C}^{n}, 0\right)$ is a nilpotent group then the solubility length of $G$ satisfies $l(G) \leq n$. 
The general case $\left(G \subset \operatorname{Diff}\left(\mathbb{C}^{n}, 0\right)\right)$ is true, see (Martelo and Ribon in press). In the following example, we give a solvable group of formal diffeomorphisms tangent to the identity which is not metabelian. This contradicts Corollary 4.2 to Proposition 4.1 in (Martinez 2003).

Example 0.9. Consider the subgroup $G \subset$ Diff $_{\text {Id }}$ $\left(\mathbb{C}^{2}, 0\right)$, which is solvable but not metabelian, given by $G=\langle(\hat{h}(x), \hat{\mathrm{a}}(x)+\hat{b}(x) \mathrm{y}) ; \hat{h} \in H\rangle$, where $H$ $\subset$ Diff $_{\text {Id }}(\mathbb{C}, 0)$ is any metabelian tangent to the identity subgroup, $\hat{a}(x) \in \mathbb{C}[[x]]$ has order greater than 2 and $\hat{b}(x) \in \mathbb{C}[[x]]$ is a unit, $\hat{b}(0)=1$.

We also obtain the following description of abelian groups tangent to the identity:

Theorem 0.10. Let $G \subset \operatorname{Diff}\left(\mathbb{C}^{n}, 0\right)$ be an abelian subgroup tangent to the identity, with associate Lie algebra $\mathfrak{g}$. Then the possibilities are:

1. There are $\hat{X} 1, \ldots, \hat{X}_{n} \in \mathfrak{g}$ such that $\left[\hat{X}_{j}, \hat{X}_{r}\right]=$ $0, \forall i, j$ and $G \subset\left\langle\exp \left(t_{1} \hat{X}_{1}\right) \circ \cdots \circ \exp \left(t_{n} \hat{X}_{n}\right)\right|$ $t j \in \mathbb{C}\rangle$.

2. There are $\hat{X}_{1}, \ldots, \hat{X}_{1} \in \mathfrak{g}$, for some $l \in\{1, \ldots$, $n-1\}$, such that $\left[\hat{X}_{j}, \hat{X}_{r}\right]=0, \forall i, j$ and $G \subset$ $\left\langle\exp \left(u_{1} \hat{X}_{1}\right) \circ \cdots \circ \exp \left(u_{l} \hat{X}_{l}\right)\right| u_{j} \in K_{n}, \hat{X}_{r}\left(u_{j}\right)$ $=0, \forall 1 \leq r, j \leq l\rangle$.

In particular, for $n=2$ we have the following possibilities:

(i) G leaves invariant an exact rational one-form, say $\hat{\omega}=d T$ for some rational function $T \in K_{2}$.

(ii) $G$ embeds into the flow of a formal vector field $\hat{X} \in \log (G)$.

(iii) There are two invariant linearly independent commuting formal vector fields $\hat{X}, \hat{Y} \in \log (G):=\{\hat{X}$ $\left.\in \hat{X}\left(\mathbb{C}^{n}, 0\right) / \exp (\hat{X}) \in G\right\}$.

\section{ABELIAN GROUPS AND CLOSED ONE-FORMS}

Before stating our next main results we observe that in some main applications of the results in Theorem 0.6 (case $\mathrm{n}=1$ ), an interesting approach consists of constructing, from the information on the holonomy groups of the foliation, some suitable differential forms which allow to "integrate" the foliation (e.g. a foliation admitting a Liouvillian first integral), see (Camacho and Scárdua 2001) for instance. More precisely, in dimension $n=1$ a formal vector field $\hat{X} \in \hat{\mathcal{X}}(\mathbb{C}, 0)$ can be written either as a linear vector field or, in case it has a zero of order $\geq 2$ at the origin, as $\hat{X}(z)=\frac{z^{k+1}}{1+\lambda_{z} k} \frac{d}{d z}$, for some $\lambda \in \mathbb{C}$ and $k \in \mathbb{N}$. Let us focus on the nonlinear case. The duality equation $\hat{\omega} \cdot \hat{X}=1$ has, in this dimension one case, a single solution:

$$
\hat{\omega}=\lambda \frac{d z}{z}+\frac{d z}{z^{k+1}}
$$

moreover, given a formal diffeomorphism $\hat{g} \in$ Diff $(\mathbb{C}, 0)$ we have:

$$
\begin{aligned}
& \hat{g}_{*} \hat{X}=\hat{X} \Longleftrightarrow \hat{g}^{*} \hat{\omega}=\hat{\omega}, \hat{g}_{*} \hat{X}=c_{\hat{g}} \hat{X} \\
& \text { for some } c_{\hat{g}} \in \mathbb{C}^{*} \Longleftrightarrow g^{*} \hat{\omega}=\frac{1}{c_{\hat{g}}} \hat{\omega} .
\end{aligned}
$$

Equation (1) is the general expression of a closed meromorphic one-form with an isolated pole of order $k+1$ at the origin $0 \in \mathrm{C}$, residue $\lambda$, in a suitable coordinate system. It is a particular case of the Integration Lemma ((Cerveau and Mattei 1982), (Scárdua 1997) Example 1.6 page 174). Finally, notice that in dimension-one each formal or meromorphic one-form is closed. This suggests, in view of Theorem 0.6 and all the above, that one may expect to obtain results relating algebraic properties of subgroups of $\operatorname{Diff}\left(\mathbb{C}^{n}, 0\right)$ with the existence of suitable closed one-forms. Therefore, one necessary tool is a (natural) version of the Integration Lemma to the case of formal one-forms.

We shall see (cf. Theorem 0.11 below) that a subgroup $G \subset \operatorname{Diff}\left(\mathbb{C}^{2}, 0\right)$ admitting two commuting formal invariant vector fields, exhibits two independent invariant closed formal meromorphic one-forms. A further investigation of this situation involves the notion of formal separatrix, which is set naturally in terms of formal curves, i.e., equivalence 
classes in the local ring $\hat{\mathcal{O}}_{2}$, under the following equivalence relation: given $\hat{\varphi}, \hat{\psi} \in \mathcal{O}_{2}$ we have $\hat{\varphi} \sim$ $\hat{\psi} \Leftarrow \hat{\varphi}=\hat{u} . \hat{\psi}$ for some unit $\hat{u} \in \hat{\mathcal{O}}_{2}$. A formal curve $[\varphi]$ will be called a separatrix of a subgroup $G \subset \mathrm{D}$ $\hat{i f f}\left(\mathbb{C}^{2}, 0\right)$ if it is invariant by each element of this group in the obvious sense. Two formal curves $[\hat{\varphi}]$ and $[\hat{\psi}]$ are called transverse if each tangent space has dimension one and the tangent spaces span $\mathbb{C}^{2}$. As a converse of (iii) in Theorem 0.10 we have:

Theorem 0.11. Let $G \subset \operatorname{Diff}\left(\mathbb{C}^{2}, 0\right)$ be a subgroup admitting two linearly independent invariant commuting formal vector fields. Then $G$ admits two closed, independent, formal meromorphic, invariant one-forms. The group $G$ is abelian provided that one of the following conditions is satisfied:

\section{1. $G$ is tangent to the identity.}

\section{G exhibits two formal transverse separatrices.}

The notions of formal closed meromorphic oneform and other formal objects are stated naturally in terms of formal expressions having coefficients that belong to the field $\hat{K}_{n}$. In (2) there is a natural relation between the polar set of the invariant forms and the separatrices of the the group. Since the polar set of an invariant form is also invariant by the group, it must be a union of separatrices of the group. Using this, the second part of Theorem 0.11 and the abovementioned formal Integration Lemma, we obtain normal forms for abelian subgroups admitting two transverse separatrices and having Lie algebra of dimension two, cf. (Martelo and Scárdua 2011).

\section{Metabelian Groups}

The above is concerned with the abelian case. For the metabelian non-abelian case we have:

Theorem 0.12. Let $G \subset \operatorname{Diff}\left(\mathbb{C}^{2}, 0\right)$ be $a$ metabelian non-abelian subgroup. Assume that the group of commutators $G^{(1)}=[G, G]$ is tangent to the identity, for instance if the linear part group $D G \subset \mathrm{GL}(2, \mathbb{C})$ is abelian. Denote by $\mathfrak{g}([G, G])$ the associate Lie algebra of $[G, G]$. We have the following possibilities: (i) $\mathfrak{g}([G, G])$ is one-dimensional. There is a formal vector field $\hat{X}$ with $\exp \hat{X} \in G$, such that, for each $\hat{g} \in G$ there is a rational function $T_{\hat{g}} \in \hat{K}_{2}$ that satisfies: $\hat{X}\left(T_{\hat{g}}\right)=0$ and $\hat{g}^{*}(\hat{X})=T_{\hat{g}} \cdot \hat{X}$.

(ii) $\mathfrak{g}([G, G])$ is two-dimensional. There are two C-linearly independent formal vector fields $\hat{X}, \hat{Y}$ $\in g=\log G$, such that (ii.1) $[\hat{X}, \hat{Y}]=0$. (ii.2) For each $\hat{g} \in G$ there are $\left(s_{1}, t_{1}\right),\left(s_{2}, t_{2}\right) \in \mathbb{C}^{2}$ linearly independent that satisfy $\hat{g}^{*}(\hat{X})=s_{1} \hat{X}+t_{1} \hat{Y}$ and $\hat{g}=$ $(\hat{Y})=s_{2} \hat{X}+t_{2} \hat{Y}$

Furthermore, in this last case there are two $\mathbb{C}$-linearly independent closed formal meromorphic one-forms $\hat{\omega}_{j},(j=1,2)$ and $a_{j}, b_{j} \in \mathbb{C}^{*}$ such that $\hat{g}$ $*\left(\hat{\omega}_{j}\right)=\mathrm{a}_{j} \hat{\omega}_{1}+b_{j} \hat{\omega}_{2}, \forall \hat{g} \in G$.

Groups as in (ii.2) above are described in (Martelo and Scárdua 2011).

\section{GROUPS CONTAINING DICRITIC DIFFEOMORPHISMS}

Now we consider groups of formal diffeomorphisms under the hypothesis of the existence of a suitable dicritic (radial type) element. More precisely, according to (Martinez 2003), a diffeomorphism $\hat{f} \in \operatorname{Diff}_{k}\left(\mathbb{C}^{n}, 0\right)$ is called dicritic if $\hat{f}(z)=z+\hat{f}$ ${ }_{k+1}(z)+\hat{f}_{k+2}(z)+\cdots$, where $\hat{f}_{k+1}(z)=f(z) z$ and $\mathrm{f}$ is a homogeneous polynomial of degree $k$. A formal vector field $\hat{X} \in \hat{\mathcal{X}}_{k}\left(\mathbb{C}^{n}, 0\right), k \geq 1$ is called dicritic if $\hat{X}=f(z) \vec{R}+\left(\mathrm{p}_{k+2}^{(1)}+\cdots\right) \frac{\partial}{\partial z_{1}}+\cdots+\left(\mathrm{p}_{k+2}^{(n)}+\cdot \cdot\right) \frac{\partial}{\partial z_{n}}$ where $\mathrm{f} v$ is a homogeneous polynomial of degree $v$, and $\mathrm{f}$ is homogeneous of degree $k$, and $\vec{R}=z_{1} \frac{\partial}{\partial z_{1}}+$ $\cdots+z_{n} \frac{\partial}{\partial z_{n}}$ is the radial vector field.

Definition 0.13 (Regular dicritic vector fields and diffeomorphisms). We also introduce the following useful subclasses of dicritic diffeomorphisms and vector fields. A formal vector field $\hat{\mathcal{X}}_{k} \ni \hat{X}=f(z) \vec{R}+\left(\mathrm{p}_{k+2}^{(1)}+\cdots\right) \frac{\partial}{\partial z_{1}}+\cdots+\left(\mathrm{p}_{k+2}^{(n)}+\right.$ ...) $\frac{\partial}{\partial z_{n}}$ is called regular dicritic if $\hat{X}$ is dicritic and there are $i_{0}, j_{0} \in\{1, \ldots, n\}$, such that $f$ and $z_{j 0} \mathrm{p}_{k+2}^{\left(i_{0}\right)}$ $z_{i 0} \mathrm{p}_{k+2}^{\left(j_{0}\right)}$ are coprime. This implies that 0 is an isolated singularity of $\hat{X}$. A formal diffeomorphism $\hat{f} \in \mathrm{D} \hat{\mathrm{i}}$ $\mathrm{ff}_{k}\left(\mathbb{C}^{n}, 0\right)$ is called regular dicritic if its infinitesimal generator is a regular dicritic vector field. 
We have, analogously to Theorem 0.6 , for groups containing a regular dicritic element.

Theorem 0.14. For a subgroup $G \subset \operatorname{Diff}\left(\mathbb{C}^{n}, 0\right)$ containing a regular dicritic diffeomorphism, the following conditions are equivalent:

1. $G \cap \operatorname{Diff}_{\text {Id }}\left(\mathbb{C}^{n}, 0\right)$ is abelian.

2. $G$ admits a projectively invariant regular dicritic formal vector field.

In particular, a subgroup of $\mathrm{Diff}\left(\mathbb{C}^{n}, 0\right)$ tangent to the identity and containing a regular dicritic diffeomorphism is abelian if and only if it admits an invariant formal vector field.

Regarding the case of metabelian groups we have:

Theorem 0.15. A subgroup of formal diffeomorphisms containing a regular dicritic diffeomorphism and with abelian linear part group is metabelian provided that it admits a projectively invariant formal vector field.

As a partial converse of Theorem 0.15 we have:

Proposition 0.16. Let $G \subset \operatorname{Diff}\left(\mathbb{C}^{n}, 0\right)$ be $a$ metabelian subgroup containing a regular dicritic diffeomorphism with order of tangency $k$. Suppose that $D G$ is abelian and there is a linear diffeomorphism $\hat{h}$ $\in G$, given by $\hat{h}(z)=\lambda \cdot \mathrm{Id}$, where $\lambda \in \mathbb{C}$ is such that $\lambda^{\mathrm{k}} \neq 1, \lambda^{\mathrm{k}+1} \neq 1$. Then there is a formal vector field $\hat{X} \in \hat{\mathcal{X}}_{j}\left(\mathbb{C}^{n}, 0\right), j \geq 2$, which is projectively invariant by $G$. In particular, given a subgroup $G=\langle\hat{f}, \hat{h}\rangle$ $\subset \operatorname{Diff}\left(\mathbb{C}^{n}, 0\right)$, where $f^{n}$ is regular dicritic and $\hat{h}(z)=$ $\lambda z, \lambda^{\mathrm{k}} \neq 1, \lambda^{\mathrm{k}+1} \neq 1$. Then the group $G$ is metabelian if and only if $\left[\hat{f}, \hat{h}^{2}\right]$ and $\left[\hat{f}^{2}, \hat{h}\right]$ commute with $[\hat{f}, \hat{h}]$.

Next we state an equivalence similar to the dimension one case, but for groups that contain some dicritic diffeomorphism.

Theorem 0.17. For a subgroup $G \subset \mathrm{Diff}_{\text {Id }}\left(\mathbb{C}^{n}, 0\right)$ of formal diffeomorphisms tangent to the identity and containing a dicritic diffeomorphism tangent to the identity with order $k$, the following statements are equivalent:
(1) The group is abelian.

(2) The group is nilpotent.

(3) Every non-trivial element in the group is tangent to the identity with order $k$.

Our results apply to the study of foliations on complex projective spaces and other ambient manifolds as well. The class of singularities which correspond, via the holonomy of its separatrices, to the class of regular dicritic diffeomorphisms is to be formally introduced and studied in a forthcoming work. Using an adaptation of a classical result due to Hironaka and Matsumara (Hironaka and Matsumara 1968) we may be able to move from the "formal world" (considered is this announcement) to the "analytic/convergent world", which is the natural ambient to the study of holomorphic foliations with singularities.

A final word should be said about the possible applications of our results. We are interested in the study of Liouvillian integration for holomorphic foliations of codimension $n \geq$ 1. As suggested by the codimension 1 cases see for instance (Scárdua 2011), this passes through the comprehension of algebraic, geometric and formal structures of subgroups of $\operatorname{Diff}\left(\mathbb{C}^{n}, 0\right)$ in terms we propose in this work.

\section{RESUMO}

Nesta nota anunciamos alguns resultados no estudo de grupos de difeomorfismos formais e germes de difeomorfismos em várias variáveis complexas. Tais grupos estão relacionados com o estudo de estruturas transversais e a dinâmica das folheações holomorfas, através da noção de grupo de holonomia da folha de uma folheação. Para dimensão um, há um amplo dicionário relacionando classificação analítica/formal do grupo, com suas propriedades algébricas (finitude, comutatividade, solubilidade, dentre outras). Tal sistema de equivalências também caracteriza a existência de certos fatores de integração, ou seja, campos de vetores e one-forms diferenciais invariantes associados ao grupo. Nosso objetivo é estabelecer as linhas básicas de um tal dicionário 
para o caso de grupos em várias variáveis complexas. Nossos resultados podem ser aplicados na construção de certos fatores de integração para folheações holomofas singulares. Acreditamos que estes podem representar um ponto de partida no estudo da conexão entre Integração Liouvilliana e estruturas transversais de folheações holomorfas singulares no caso de codimensão arbitrária. Os resultados nesta comunicação são derivados da tese de Doutorado "Grupos de germes de difeomorfismos complexos em várias variáveis e formas diferenciais" do primeiro autor (Martelo 2010).

Palavras-chave: Difeomorfismo formal complexo, gerador infinitesimal, folheação holomorfa, germe de difeomorfismo complexo.

\section{REFERENCES}

CAmacho C AND Lins Neto A. 1985. Geometry theory of foliations. Translated from the Portuguese by Sue E. Goodman. Birkhauser Boston, Inc., Boston, MA.

CAMACho C, Lins Neto A AND SAD P. 1992. Foliations with algebraic limit sets. Ann Math 136: 429-446.

CAMACHO C AND SCÁRdUA B. 2001. Holomorphic foliations with Liouvillian first integrals. Ergod Theor Dyn Syst 21(3): 717-756.

Cerveau D AND Mattei JF. 1982. Formes intégrables holomorphes singulières. Astérisque 97.

EISENBUD D. 1995. Commutative algebra with a view toward algebraic geometry. Graduate Texts in Mathematics, Springer-Verlag, New York 150.

HIRONAKA H AND MATSUMARA H. 1968. Formal functions and formal embeddings. J Math Soc JPN 20: 1-2.
II'YASHENKO Y. 1978. Topology of phase portraits of analytic differential equations on a complex projective plane. Trudy Sem Petrovsk 4: 83-136.

II'YASHENKO Y AND YAKOVENKO S. 2007. Lectures on analytic differential equations. Graduate studies in mathematics. American Mathematical Society 86.

LORAY F. 1994. Feuilletages holomorphes à holonomie résoluble. These Université de Rennes I.

Martelo M. 2010. Grupos de germes de difeomorfismos complexos em várias variáveis e formas diferenciais. $\mathrm{PhD}$. Thesis, Instituto de Matemática, Universidade Federal do Rio de Janeiro.

MARTELO MAND RIBON J. IN PRESS. Derived length of solvable groups of local diffeomorphisms. Math DS, arXiv: $1108.5779 \mathrm{v} 1$

MARTELO M AND SCÁRDUA B. 2011. Integrating factors for groups of formal complex diffeomorphisms. Math CV. Available at arXiv. arXiv:1110.5786v1

MARTINEZ FB. 2003. Groups of germs of analytic diffeomorphisms in (C2, 0). J Dyn Control Syst, New York 9(1): 1-32.

NAKAI I. 1994. Separatrices for non-solvable dynamics on (C, 0). Annales de l'institut Fourier (Grenoble) 44(2): 569-599.

SCÁRDUA B. 1997. Transversely affine and transversely projective foliations. Annales Scientifiques de l'École Normale Supérieure 30(4): 169-204.

SCÁRDUAB. 1999. Integration of complex differential equations. J Dyn Control Syst New York 5: 1-50.

SCÁRDUA B. 2011. Differential Algebra and Liouvillian first integrals of foliations. J Pure and Appl Algebra 215(5): 764-788.

SCHERBAKOV AA. 2006. Dynamics of Local Groups of Conformal Mappings and Generic Properties of Differential Equations on $\mathbb{C}^{2}$. Tr. Mat. Inst. im. V.A. Steklova, Ross. Akad. Nauk 254: 111-129. [Proc Steklov Inst Math 254: 103-120]. 